\title{
TEMA 14-2015:Endocarditis Infecciosa
}

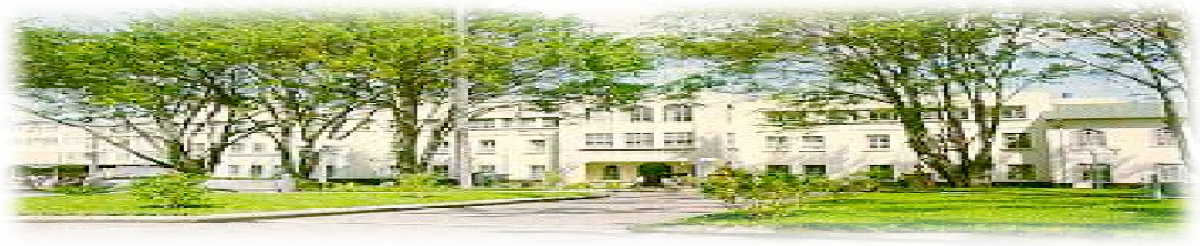

Hospital San Juan de Dios, San José, Costa Rica. Fundado en 1845

Recibido:

Aceptado:
$26 / 05 / 2015$

$30 / 08 / 2015$

Benjamín Ramírez Cisneros ${ }^{1}$

Giovanni Sedó Mejía ${ }^{2}$

Ericka Hütt Centeno ${ }^{3}$

\footnotetext{
${ }^{1}$ Médico General. Universidad de Costa Rica Hospital San Juan de Dios. benramcis@gmail.com

${ }^{2}$ Médico Especialista en Medicina Interna y Alergología, Medicina 4. Hospital San Juan de Dios. Profesor de la Cátedra de Medicina Interna UCR.

${ }^{3}$ Médico General. Universidad de Costa Rica, Hospital San Juan de Dios.
}

\section{RESUMEN}

La endocarditis infecciosa es una enfermedad con elevada morbimortalidad. Afecta principalmente a personas con cardiopatías degenerativas, adultos mayores y antecedente de cardiopatía reumática. El endotelio valvular o endocárdico es naturalmente resistente a la colonización de bacterias, por lo que debe de haber una lesión y formación de trombo que es luego colonizado por microorganismos, generando lo que se conoce como una vegetación. Los agentes causales más aislados incluyen los estreptococos orales y los estafilococos. La mayoría de pacientes se presenta con fiebre, síntomas constitucionales y un soplo de novo o que cambió de características. Los criterios modifi-cados de Duke incluyen criterios clínicos, microbiológicos y ecocardiográficos. Se recomienda a todo paciente con sospecha moderada de endocarditis infecciosa, realizar inicialmente un ecocardiograma transtorácico. Las complicaciones cardiacas son las más comunes, seguidas por las complicaciones neurológicas. Hoy en día se aboga por un tratamiento quirúrgico temprano, sin embargo, hasta un $25 \%$ de los pacientes quirúrgicos no se operan. La piedra angular del tratamiento son los antibióticos bactericidas parenterales y se tiende a utilizar terapia doble con acción sinergista.

\section{PALABRAS CLAVE}

Endocarditis infecciosa, ecocardiograma, Staphylococcus aureus.

\section{ABSTRACT}

Infective endocarditis is a disease with an elevated morbimortality. It affects mainly patients with degenerative cardiopathy, elderly patients and patients who have suffered rheumatic fever. The valvular and endocardic endothelium is 
naturally resistant to the colonization of bacteria, hence there has to be an injury and formation of a thrombus that is later colonized by microorganisms, this generates what is known as vegetation. The two main groups of bacteria isolated from bloodcultures are oral Streptococcus and Staphylococcus. The majority of patients present themselves with fever, constitutional symptoms and a new murmur or a murmur that has changed of characteristics. The modified Duke criteria include clinical, microbiological and echocardiographic criteria. It's advised to perform initially a transthoracic echocardiogram to all patients with a moderate probability to have infective endocarditis. The cardiac complications are the most common, followed by neurologic complications. The new tendency is for patients to be treated with surgery at early stages of the disease, even though, up to $25 \%$ of patients with surgical indications arenot operated. The cornerstone of the treatment is parental antibiotic, usually therapy is dual and the two agents used have synergetic properties.

\section{KEY WORDS}

Infectious endocarditis, echocardiogram, Staphylococcus aureus.

\section{INTRODUCCIÓN}

La endocarditis es la inflamación del recubrimiento interno del corazón (endocardio) y las válvulas cardiacas. Los agentes infecciosos son los principales responsables de dicha entidad. ${ }^{(1)}$. La endocarditis infecciosa (EI) es una enfermedad grave y, pese a grandes avances en procedimientos diagnósticos y terapéuticos, continúa siendo una enfermedad con alta morbimortalidad. ${ }^{2}$ Como síndrome infeccioso, es una entidad sumamente heterogénea, con una clasificación compleja, que varía según si hay patología cardiaca subyacente, el agente microbiológico $\mathrm{y}$ localización de la infección. ${ }^{(2)}$

La presentación clínica de la EI ha cambiado, principalmente en países desarrollados, donde hay un mayor reporte de EI asociado a cuidados de salud (siendo algunos casos nosocomiales) ${ }^{3}{ }^{(3)}$ Al ser una enfermedad cuya incidencia aumenta con la edad ${ }^{(4)} 4 \mathrm{y}$ al tener Costa Rica una expectativa de vida de país desarrollado, será una enfermedad a la cual nos tendremos que enfrentar. Es una entidad clínica potencialmente fatal, que se debe reconocer, diagnosticar y tratar tempranamente para disminuir morbimortalidad. ${ }^{(4)}$

\section{Epidemiología y factores de riesgo}

La epidemiología de la EI ha cambiado en las últimas décadas, sobretodo en países desarrolla$\operatorname{dos}^{2}{ }^{(2)}$ Inicialmente se identificó dicho síndrome como una entidad que afectaba a adultos jóvenes portadores de valvulopatías, en su gran mayoría secundaria a fiebre reumática. ${ }^{(2)}$ Actualmente los factores de riesgo más notables incluyen: valvulopatía degenerativa, prolapso de válvula mitral4, ${ }^{(4)}$ pacientes portadores de válvulas protésicas o aparatos intracardiacos (como marcapasos) y consumidores de drogas intravenosas (IV). ${ }^{(3)}$ En Costa Rica se identificó a las cardiopatías estructurales como el principal factor de riesgo para desarrollar EI; en nuestro país la cardiopatía reumática aún es un factor de riesgo frecuente. ${ }^{(5)}$ En la población pediátrica, los tres factores de riesgo más identificados en Costa Rica fueron cardiopatías congénitas, uso de catéter intravascular y desnutrición. ${ }^{(6)}$

El hecho de que varios de estos factores de riesgo se confabulen en adultos mayores, es lo que explica la alta incidencia en este grupo. Más del $50 \%$ de los casos de EI en los Estados Unidos se dan en pacientes mayores de 60 años. ${ }^{(4)}$ Se estipula que este cambio de paradigma en la epidemiología de EI se debe a un aumento de comorbilidades y más exposición a centros de salud. ${ }^{(7)}$

La EI tiene una incidencia anual de 3 a 9 casos por 100000 habitantes en países industrializados. ${ }^{(7)} \mathrm{La}$ American Heart Association (AHA) estima entre 100000 a 200000 casos nuevos anuales en Estados Unidos por año. ${ }^{(7)}$ Un estudio nacional ${ }^{(5)}$ estimó una frecuencia de 1,79 por 100000 pacientes/año ingresados en un hospital de referencia nacional. La literatura reporta que es de dos a tres veces más frecuente en el género masculino por razones desconocidas ${ }^{(4)}$, en Costa Rica se ha reportado el mismo comportamien- 
to. ${ }^{(5)}$ Recientemente han aumentado los casos de EI asociados al cuidado de salud (nosocomiales y no nosocomiales), en Estados Unidos se reportó más de un tercio de los casos totales como casos asociados al cuidado de salud. ${ }^{(3)}$

\section{Clasificación}

La EI antiguamente era clasificada por su presentación clínica, en aguda (usualmente causada por Staphylococcus), subaguda (usualmente causada por Streptococcus) y crónica. ${ }^{(3)}$ Actualmente se clasifica según su localización y presencia de material intracardiaco, modo de adquisición, actividad o recurrencia ${ }^{2}{ }^{(2)}$ En el cuadro 1 se describe la clasificación y sus definiciones con mayor detalles.

\section{Fisiopatología}

El endotelio valvular es naturalmente resistente a la colonización de bacterias ${ }^{(3)}$; fisiopatológicamente existe una premisa de que debe de existir un daño endotelial para que inicie la colonización bacteriana. El primer evento es la adherencia del patógeno al coágulo formado, que implica la presencia de bacteremia, luego se da la proliferación de la bacteria en la lesión cardiaca y puede conllevar extensión local con daño de tejido circundante o lesiones embólicas. ${ }^{(8)}$ Actividades cotidianas como masticar comida y cepillarse los dientes son capaces de producir bacteremias frecuentes, transitorias y de baja carga ${ }^{(1)}$, suficientes como para colonizar el endocardio. Esto probablemente explica porqué la mayoría de casos de EI no está precedida por procedimientos médico-quirúrgicos que están asociados a bacteremias de mayor grado. ${ }^{(8)}$

El daño endotelial previamente descrito puede resultar de lesiones por el flujo sanguíneo turbulento en pacientes con valvulopatías, por electrodos, catéteres, aparatos intracardiacos o pequeñas partículas que son inoculadas al utilizar drogas intravenosas. ${ }^{(3)} \mathrm{La}$ inflamación crónica, que se observa en la cardiopatía reumática crónica y las lesiones valvulares degenerativas, también pueden desencadenar una EI. ${ }^{(3)}$

Cuadro I. Clasificación y definición de endocarditis infecciosa

Localización y presencia de material intracardiaco

- Izquierda, válvula nativa

- Izquierda, válvula prostética

- Temprana $<1$ año después de la cirugía

- Tardía > 1 año después de la cirugía

- Derecha

- Relacionada con material intracardiaco (como marcapaso)

\section{Modo de adquisición}

- Asociado al cuidado de salud

- Nosocomial: EI en un paciente hospitalizado $>48 \mathrm{~h}$ antes del inicio de signos y síntomas consistentes con EI.

- No nosocomial: Signos y síntomas de EI inician en las primeras $48 \mathrm{~h}$ de ingreso en un paciente asociado al cuidado de salud

- Enfermería en casa, terapia intravenosa domiciliar, hemodiálisis, quimioterapia intravenosa $<30$ días previo al inicio de EI.

Hospitalizado en un centro de salud < 90 días previo al inicio de EI.

- Habitante de un centro de cuido.

- Adquirido en la comunidad: Signos y síntomas de EI inician $<48 \mathrm{~h}$ del ingreso en un paciente que no cumple criterios para considerarse asociado al cuidado de salud

- Asociado al uso de drogas de abuso intravenosas: EI en un abusador activo de drogas IV sin fuente alternativa de infección.

\section{Endocarditis infecciosa activa}

- Fiebre persistente y hemocultivos positivos o

- Morfología de inflamación activa encontrada en cirugía o

- Pacientes aún con antibioticoterapia o

- Evidencia histopatológica de EI activa

\section{Recurrencia}

- Recaída: episodios repetidos de EI causados por el mismo microorganismos $<6$ meses después del episodio inicial

- Reinfección: infección con microorganismo distinto o episodio repetido de EI causado por el mismo microorganismo $>6$ meses después del primer episodio.

Fuente: Adaptado de European Heart Journal 2009; 30: 23692413. 
Al haber lesión endotelial, se ponen en contacto la sangre con los componentes subendoteliales, por definición procoagulantes, por lo que se produce un pequeño coágulo. ${ }^{(9)}$ Algunos de estos componentes son proteínas de la matriz extracelular, tromboplastina y factor tisular. ${ }^{(8)}$ Los patógenos asociados a la EI, al estar circulando en sangre, se unen a estas estructuras y colonizan el coágulo. Estas bacterias atraen células inflamatorias, como monocitos ${ }^{(99)}$, que a su vez secretan citoquinas y otros factores procoagulantes que contribuyen a que el coágulo infectado crezca; dicho coágulo infectado es lo que se conoce como una vegetación. ${ }^{(8)}$

Las bacterias asociadas a EI se unen al coágulo mediante proteínas ligadoras a fibronectina $\mathrm{y}$ adhesinas conocidas como moléculas adhesivas de la matriz que reaccionan con compuestos de la superficie del microorganismo MSCRAMM (por sus siglas en inglés). ${ }^{(8)}$ El $S$. aureus es el microorganismo del que más se ha estudiado este mecanismo de adhesión al coágulo.

Una vez que colonizan el endocardio y el coágulo, se llevan a cabo mecanismos para escapar las defensas del huésped, un evento clave para este proceso es la maduración de la vegetación. ${ }^{(8)} \mathrm{El}$ proceso de maduración también conlleva el depósito de fibrina, plaquetas y proliferación bacteriana en el coágulo inicialmente estéril1. ${ }^{(1)}$ En este proceso el coágulo es completamente invadido por los microorganismos y estos se recubren por el mismo para evadir la respuesta inmunitaria del huésped. ${ }^{(8)}$ Histológicamente, la vegetación está conformada por fibrina, plaquetas y bacterias, al no tener vasos sanguíneos hace difícil la penetración de células fagocíticas $1^{(1)} \mathrm{Se}$ ha demostrado que estas bacterias son resistentes a las proteínas microbicidas plaquetarias, por lo que las estas inducen activación plaquetaria y su efecto procoagulante, que hace madurar la vegetación, sin ser eliminadas. ${ }^{(8)}$

Este modelo fisiopatológico explica principalmente la patogénesis de bacterias gram positivas, pero no explica de forma adecuada la fisiopatología para microorganismos intracelulares. ${ }^{(3)}$
La EI es más frecuentemente producida por bacterias gram positivas; se estipula que esta diferencia se debe a los mecanismos de adherencia al coágulo y por diferencias en la susceptibilidad a ser eliminadas en la sangre. ${ }^{(8)}$ El complejo de ataque de membrana del complemento es muy efectivo para matar bacterias gram negativas, pero las bacterias gram positivas son naturalmente resistentes. ${ }^{(8)}$ La EI no es una enfermedad que responde ante los mecanismos de defensa inmunológicos del huésped, lo que explica porque el tratamiento antibiótico se basa en antibioticoterapia bactericida parenteral. ${ }^{\left({ }^{2}\right)}$

\section{Microbiología}

Numerosos organismos están relacionados con la EI; los más importantes son los agentes gram positivos Estafilococos y Estreptococos. ${ }^{(4)} \mathrm{El}$ perfil microbiológico ha cambiado en los últimos años, y la mayoría de la literatura actualmente reporta que los Estafilococos son los agentes causales más frecuentes. ${ }^{(3)}$ Los agentes causales más importantes son $S$. aureus,Estreptococos del grupo viridans (u orales), Enterococos, Estafilococos coagulasa-negativos, Streptococcus bovis y el grupo de bacterias gram negativas conocidas HACEK (Haemophilus parainfluenzae, Haemophilus aphrophilus, Haemophilus oaraphrophilus, Actinobacillus actinomycetemcomitans, Cardiobacterium hominis, Eikenella corrodens y Kingella spp.). ${ }^{(4)}$ En Costa Rica se identificó que los Estreptococos orales seguido por $S$. aureus son los agentes etiológicos más aislados. ${ }^{(5)}$ En la población pediátrica el agente causal más frecuentemente aislado es el $S$. aureus. ${ }^{(5)}$

La literatura reporta que el $90 \%$ de los hemocultivos en los pacientes con EI son positivos ${ }^{(3,8)}$, aislándose en el 80-85\% Estafilococos o Estreptococos. ${ }^{(2,3)}$ En Costa Rica se reportó que un $80 \%$ de los hemocultivos presentó al menos un hemocultivo positivo, mientras que un $70 \%$ los dos hemocultivos. ${ }^{(5)}$ El $10 \%$ de hemocultivos que se reportan negativos se podría deber a que el paciente recibió antibióticos previamente o que se trate de algún microorganismo fastidioso, es decir difícil de cultivar. ${ }^{(3)}$ Recientemente ha au- 
mentado el número de técnicas diagnósticas que permiten aislar con mayor frecuencia estos gérmenes, como Coxiella burnetti, Brucella spp, Bartonella spp, las bacterias del grupo HACEK, Chlamydia spp., Mycoplasma spp., Legionella pnemophila y Aspergillus spp. ${ }^{(10)} \mathrm{Si}$ se realizan otras pruebas diagnósticas, se logra aislar hasta en un $60 \%$ el agente infeccioso de aquellos hemocultivos negativos. ${ }^{(3)}$

\section{Manifestaciones clínicas}

Hasta un $80-90 \%{ }^{(3,9)}$ de los pacientes se presentan con fiebre, asociada a sintomatología sistémica como escalofríos, hiporexia y pérdida de peso. ${ }^{(10)}$ Se documenta soplo hasta en $85 \%$ de los pacientes, ${ }^{(9)}$ en Costa Rica se constató un soplo cardiaco en el 91\% de los casos. ${ }^{(5)}$ En series de casos contemporáneos se han reportado un 48\% de pacientes con soplos cardiacos nuevos y un $20 \%$ de soplos cardiacos que empeoraron. ${ }^{(3)}$ Los signos clásicos periféricos como nódulos de Osler o lesiones de Janewayaún se ven en países en vías de desarrollo ${ }^{(9)}$; estos tienden a verse menos en presentaciones agudas porque la EI evoluciona muy rápido para desarrollar fenómenos vasculares inmunológicos. ${ }^{(1)}$ En Costa Rica se identificó que la fiebre seguida por la fatiga son los síntomas más comunes en pacientes con EI. ${ }^{(5)}$

Otros signos menos comunes incluyen hematuria $(25 \%)$ y esplenomegalia $(11 \%)$. El paciente puede presentarse con sepsis, meningitis, insuficiencia cardiaca, émbolos pulmonares sépticos, ictus, oclusión arterial periférica aguda e insuficiencia renal. ${ }^{(3)}$ Los reactantes de fase aguda se elevan en dos tercios de los casos, y leucocitosis y anemia se ven en aproximadamente la mitad de los pacientes. ${ }^{(3)}$ En Costa Rica se identificó que más de la mitad de los pacientes presentaban anemia $(65,5 \%)$ y leucocitosis (59\%). ${ }^{(5)}$ Un $95 \%$ de los pacientes presentó una PCR elevada. ${ }^{(5)}$

Los fenómenos clásicos, no específicos, incluyen: hemorragias en astilla ( $8 \%$ ), que son lesiones, rojizas lineares, subungueales, que no borran con la digitopresión, lesiones de Janeway (5\%), máculas, indoloras, violáceas que borran con la digitopresión en palmas y plantas, nódulos de Osler, nódulos violáceos dolorosos, que se encuentran en dedos y ortejos y manchas de Roth $(5 \%)$ y lesiones exudativas y edematosas en la retina. $^{(3,4)}$

Cuadro II. Criterios de Duke modificados

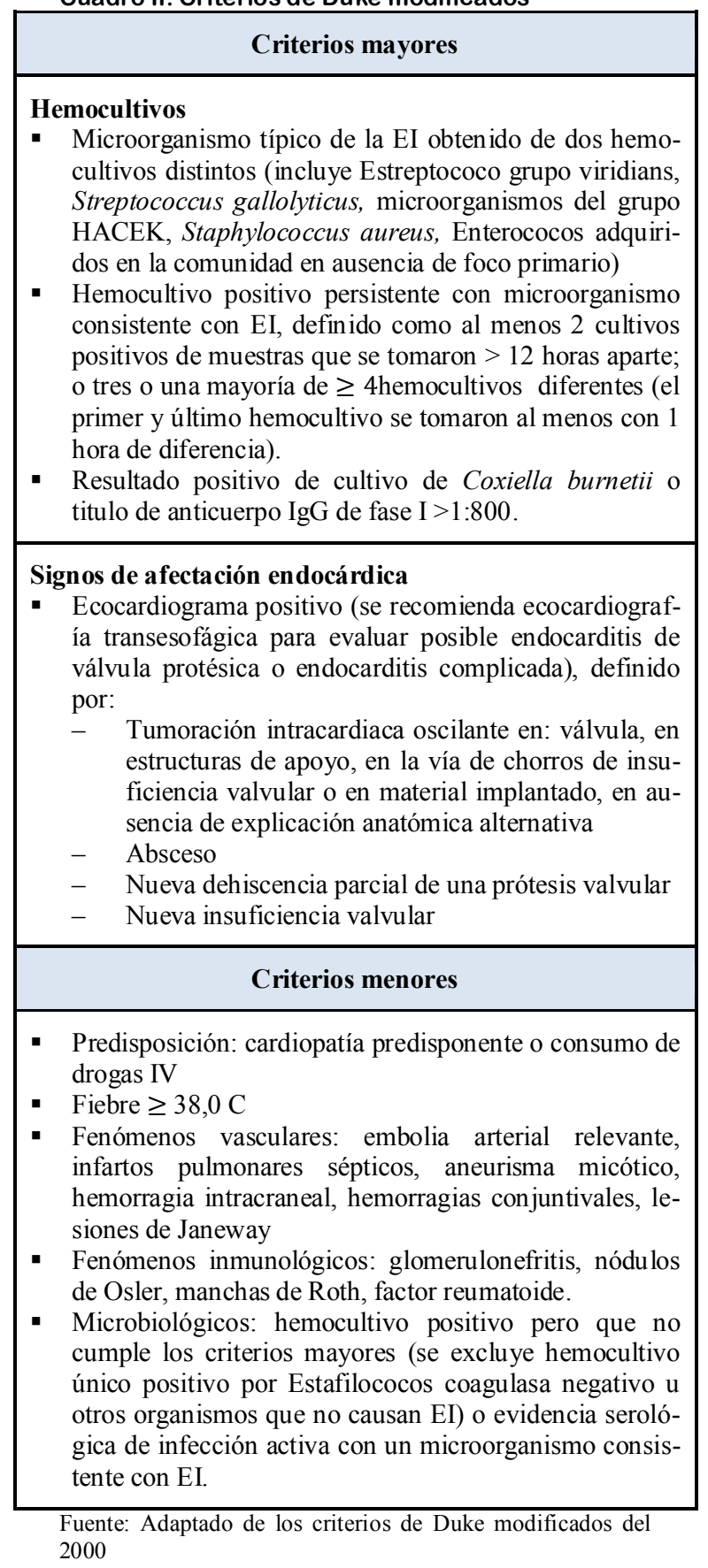




\section{Diagnóstico}

La variabilidad en la presentación clínica en este síndrome infeccioso requiere de una estrategia diagnóstica que sea sensible y específica. ${ }^{(1)} \mathrm{El}$ diagnóstico de la EI se basa en la clínica, microbiología y hallazgos ecocardiográficos. ${ }^{(3)}$ Los criterios diagnósticos de Duke, modificados en el año 2000, utilizan criterios mayores y menores ${ }^{(11)}$, con una sensibilidad y especificidad mayor al $80 \%{ }^{(3)}$ Los criterios diagnósticos de Duke se observan en el cuadro 2. La EI definitiva se define por la presencia de dos criterios mayores, un criterio mayor y tres menores o cinco criterios menores. $^{(11)}$ También se puede considerar EI definitiva por criterios patológicos: un microorganismo demostrado por cultivo o examen histológico de una vegetación (que embolizó, intracardiaca o de absceso) o lesiones patológicas como vegetación o absceso intracardiaco confirmado histología compatible con endocarditis activa. $^{(11)}$

El diagnóstico microbiológico es esencial para identificar el agente causal y poder dar antibioticoterapia con base en la prueba de sensibilidad. ${ }^{(3,4)}$ Los hemocultivos son fundamentales $y$ mandatorios en el trabajo diagnóstico de un paciente con sospecha de EI4. ${ }^{(4)}$ Se deben tomar tres juegos de hemocultivos. ${ }^{(3)}$ En caso de tratarse de un paciente agudamente enfermo, se deben tomar hemocultivos de tres venopunciones separadas durante un periodo de una hora. ${ }^{(4)}$ Más de tres a cinco hemocultivos no aumentan la probabilidad de identificar el agente causal. ${ }^{(4)}$

\section{Ecocardiograma transtorácico versus trans- esofágico}

El ecocardiograma debe ser parte del trabajo diagnóstico de un paciente con EI cuando la sospecha de la misma es al menos moderada. ${ }^{(4)}$ El ecocardiograma transtorácico (ETT) tiene una sensibilidad baja y una especificidad alta. ${ }^{(4)} \mathrm{Se}$ realiza de primero y es mejor que el transesofágico (ETE) para detectar abscesos anteriores aórticos y para valorar las consecuencias hemodinámicas de la disfunción valvular. ${ }^{(3)}$
El ETE tiene una sensibilidad y especificidad más alta y se recomienda si el resultado del ETT es negativo y aún hay una alta sospecha clínica de EI. ${ }^{(3)}$ El ETE se recomienda de entrada cuando hay una mala ventana en el ETT (paciente EPOC, obeso, con antecedente de cirugía torácica), cuando el paciente es portador de una válvula protésica o un aparato intracardiaco ${ }^{(11)}$, si se sospecha absceso, cuando hay fiebre persistente pese a antibioticoterapia adecuada o historia de abuso de drogas IV. ${ }^{(4)}$ En un estudio costarricense, por medio de ETT se identificó una vegetación en $76 \%$ de los casos y en $100 \%$ de los pacientes a los cuales se le realizó un ETE. ${ }^{(5)}$

\section{Complicaciones}

Las complicaciones cardiacas son las más comunes en pacientes con EI y ocurren en un 30 a $50 \%$ de los casos. ${ }^{(4)}$ La insuficiencia cardiaca es la causa más común de muerte y la razón más frecuente para que el paciente sea sometido a cirugía cardiaca. ${ }^{(4)}$ Otras complicaciones cardíacas incluyen abscesos locales, bloqueo del sistema de conducción, pericarditis, fístula intracardiaca o disección valvular aórtica. ${ }^{(4)}$

Las complicaciones cerebrales son las complicaciones más severas extracardiacas, además de las más frecuentes. ${ }^{(3)}$ Se dan en un 15 a $20 \%$ de los pacientes, y si se realizan estudios más específicos, como resonancia magnética, se encuentran anormalidades en hasta un $80 \%$ de los pacientes. ${ }^{(3)}$ Las complicaciones cerebrales incluyen ictus isquémico o hemorrágico, isquemia cerebral transitoria, embolismo cerebral silente, aneurisma micótico, absceso cerebral y meningitis. ${ }^{(3)}$ Es frecuente que los pacientes con EI debuten con un ictus. ${ }^{(3)}$ Otras complicaciones incluyen: infarto renal, infartos sépticos pulmonares, glomerulonefritis, osteomielitis vertebral, artritis séptica o abscesos metastásicos en riñón, bazo o tejidos blandos. ${ }^{(4)}$

\section{Tratamiento quirúrgico}


Actualmente, la EI complicada se ha convertido en una enfermedad quirúrgica. ${ }^{(10)}$ En las últimas décadas se ha abogado por el tratamiento quirúrgico temprano y las guías recomiendan que el paciente sea operado de emergencia o de urgencia. ${ }^{(10)}$ La tasa de cirugía temprana ha aumentado en aproximadamente un $50 \%$ en las últimas tres décadas. ${ }^{(3)}$ Aproximadamente la mitad de los pacientes con EI requieren de un tratamiento quirúrgico en conjunto con el uso de antibióticos. ${ }^{(12)}$

En el cuadro 3 se resumen las indicaciones quirúrgicas ${ }^{(2)}$; las principales indicaciones quirúrgicas de la EI son: insuficiencia cardiaca, infección no controlada o prevención de embolismo. $^{(2)}$

Un estudio reciente comparó el tratamiento convencional versus el tratamiento quirúrgico temprano en pacientes con EI y vegetaciones grandes. ${ }^{(13)}$ Dicho estudio demostró una reducción significativa en el punto duro primario, reduciendo la mortalidad por cualquier causa y eventos embólicos debido a una disminución en el riesgo de embolismo sistémico. ${ }^{(13)} \mathrm{Si}$ se operan a los pacientes según las indicaciones quirúrgicas establecidas por las guías internacionales, los pacientes tienen una mayor sobrevida a los 6 meses al comparar con pacientes que recibieron únicamente tratamiento antibiótico. ${ }^{(12)}$

Las tres indicaciones quirúrgicas más frecuentes en los pacientes con EI son insuficiencia valvular severa, el tamaño de la vegetación y la insuficiencia cardiaca. ${ }^{(12)}$ Se identificaron algunos predictores para que el paciente no sea considerado quirúrgico: hepatopatía crónica, ictus previo a decidir operar y agente etiológico S. aureus. ${ }^{(12)}$ El principal motivo por el cual no se haya operado a algún paciente con indicación quirúrgica fue un pobre pronóstico independientemente de la cirugía, seguido por muerte previo a cirugía, ictus, sepsis e inestabilidad hemodinámica. ${ }^{(12)}$ Hasta uno de cada cuatro pacientes que requiere cirugía no será operado. ${ }^{(12)}$

Es importante destacar que las decisiones para operar a un paciente con IE son complejas y multifactoriales, por lo que se recomienda que los pacientes con EI se manejen por equipos multidisciplinarios que incorporen internistas, infectólogos, cirujanos cardiacos y microbiólo$\operatorname{gos}^{(1)}$ y que el tratamiento sea individualizado.

Cuadro III. Recomendaciones para indicaciones de cirugía

\begin{tabular}{|c|c|}
\hline Insuficiencia cardiaca & Indicación \\
\hline $\begin{array}{l}\text { EI aórtica o mitral con insuficiencia } \\
\text { aguda severa u obstrucción de válvula } \\
\text { causando edema pulmonar refractario o } \\
\text { shock cardiogénico }\end{array}$ & Emergencia \\
\hline $\begin{array}{l}\text { EI aórtica o mitral con fístula a una } \\
\text { cámara cardiaca o al pericardio causan- } \\
\text { do edema pulmonar refractario o shock }\end{array}$ & Emergencia \\
\hline $\begin{array}{l}\text { EI aórtica o mitral con insuficiencia } \\
\text { aguda severa u obstrucción de válvula } \\
\text { y persistencia de insuficiencia cardiaca } \\
\text { o signos ecocardiográficos de pobre } \\
\text { tolerancia hemodinámica (cerrada } \\
\text { mitral temprana o hipertensión pulmo- } \\
\text { nar). }\end{array}$ & Urgencia \\
\hline $\begin{array}{l}\text { EI aórtica o mitral con insuficiencia } \\
\text { severa sin insuficiencia cardiaca }\end{array}$ & Electiva \\
\hline \multicolumn{2}{|l|}{ Infección no controlada } \\
\hline $\begin{array}{l}\text { Infección local no controlada (absceso, } \\
\text { aneurisma falso, fístula, vegetación } \\
\text { creciente) }\end{array}$ & Urgente \\
\hline $\begin{array}{l}\text { Fiebre persistente y hemocultivos } \\
\text { positivos }>7-10 \text { días. }\end{array}$ & Urgente \\
\hline $\begin{array}{l}\text { Infección causada por hongos o micro- } \\
\text { organismos multirresistentes. }\end{array}$ & $\begin{array}{l}\text { Urgen- } \\
\text { te/electiva }\end{array}$ \\
\hline \multicolumn{2}{|l|}{ Prevención de embolismo } \\
\hline $\begin{array}{l}\text { EI aórtica o mitral con vegetación } \\
\text { grande }(>10 \mathrm{~mm}) \text { seguido de uno o } \\
\text { más episodios embólicos pese a anti- } \\
\text { bioticoterapia adecuada }\end{array}$ & Urgente \\
\hline $\begin{array}{l}\text { EI aórtica o mitral con vegetación } \\
\text { grande }(10 \mathrm{~mm}) \text { y otros predictores de } \\
\text { evolución complicada (insuficiencia } \\
\text { cardiaca, absceso, infección persisten- } \\
\text { te) }\end{array}$ & Urgente \\
\hline $\begin{array}{l}\text { Vegetación muy grande aislada } \\
(>15 \mathrm{~mm}) .\end{array}$ & Urgente \\
\hline
\end{tabular}




\section{Tratamiento antibiótico}

El tratamiento antibiótico utilizado para la EI son agentes bactericidas parenterales. ${ }^{(8)}$ Las concentraciones séricas altas son deseables para asegurarse que difunda dentro de la vegetación. ${ }^{(8)}$ Más de la mitad de los pacientes con EI recibirán únicamente tratamiento antibiótico. ${ }^{(1)}$ Para infecciones de válvula nativa debido a microorganismos usuales, se da un tratamiento de dos a seis semanas. Para EI de válvula protésica se da usualmente por 6 semanas. ${ }^{(3)}$ Cuando se realice un cambio de válvula durante el tratamiento antibiótico, la duración de la antibioticoterapia debe ser igual a la de válvula nativa y no se debería de ajustar a la recomendación de válvula protésica. ${ }^{(3)}$

Las guías americanas ${ }^{(11)}$ y europeas (2) recomiendan regímenes de tratamiento antibiótico según el microorganismo y la susceptibilidad a antibióticos. En Costa Rica se identificó que un 90\% de los Estreptococos orales eran sensibles a Penicilina. ${ }^{(5)}$ En caso de los $S$. aureus solo un $46 \%$ ern sensibles a Oxacilina. ${ }^{(5)}$ Es fundamental por lo tanto identificar el agente causal por medio de hemocultivos, puesto que la terapia está basada en la prueba de sensibilidad de antibióticos del germen aislado. ${ }^{(8)}$

Debido a las características microbiológicas de la vegetación, se prefieren terapias parenterales sobre terapias orales y terapias de larga duración. ${ }^{(1)}$ Se utilizan frecuentemente los aminoglucósidos con algún antibiótico con actividad de pared (vancomicina o betalactámicos) para aprovechar el sinergismo en agentes gram positivos. $^{(1)}$

El tratamiento empírico se debe basar en si el paciente ha recibido terapia antibiótica recientemente, si la infección es en una válvula protésica o nativa y en el conocimiento de la epidemiología local y la resistencia local de los patógenos. ${ }^{(2)}$ Para válvulas nativas recomiendan utilizar ampicilina-sulbactam o amoxicilina-clavulanato más gentamicina; en caso de haber alergia a la penicilina, se puede cambiar el regimen a vancomicina, gentamicina y ciprofloxacina. ${ }^{(2)}$ En pacientes con válvula protésica y con EI temprana (menos de 12 meses desde la cirugía) se recomienda iniciar con vancomicina, gentamicina y rifampicina. ${ }^{(2)}$

\section{CONCLUSIONES}

La endocarditis infecciosa es una enfermedad con alta morbimortalidad si pasa desapercibida. La epidemiología de esta enfermedad ha cambiado en los países desarrollados en las últimas décadas, debido principalmente al aumento en la expectativa de vida y a la cantidad de pacientes que tienen aparatos intracardiacos. El endocardio tiene una resistencia natural a la colonización bacteriana, por lo que requiere de una injuria mecánica o inflamatoria para que se inicie la infección. Los microorganismos más frecuentemente relacionados con la EI son los gram positivos, y estos se aíslan hasta en $85 \%$ de los hemocultivos. Es esencial para guiar la conducta terapéutica aislar el germen, por lo que se deben de tomar hemocultivos, tempranos, previo al inicio de antibiótico.

El diagnóstico de la EI se basa en la clínica, microbiología y ecocardiográfica, por lo que se recomienda en todo paciente con sospecha al menos moderada de EI realizar un ETT inicialmente; en casos específicos se recomienda realizar de primera entrada un ETE. Las complicaciones cardiacas son las más frecuentes, seguidas de las complicaciones cerebrales, que son las más severas. Se recomienda, cuando está indicado, realizar la cirugía cardiaca tempranamente. La piedra angular de tratamiento de la EI es el tratamiento antibiótico bactericida parenteral. El tratamiento usualmente se basa en aminoglucósidos más algún antibiótico con actividad de pared, terapias de larga duración y se debe monitorizar con hemocultivos de control. La EI es un síndrome infeccioso complejo, que aún requiere mucha investigación para esclarecer dudas sobre la duración óptima de la terapia antibiótica e indicaciones quirúrgicas basadas en evidencia de mejor calidad. 


\section{BIBLIOGRAFÍA}

1. McDonald J. Acute Infective Endocarditis. Infect Dis Clin N Am. 2009;23:643-664.

2. Habit G Hoen B Tornos P et al. Guidelines on the prevention, diagnosis, and treatment of infective endocarditis (new version 2009). European Heart Journal 2009;30:2369-2413.

3. Hoen B Duval X.Infective Endocarditis. N Engl J Med 2013;368:1425-1433.

4. Paterick TE Paterick TJ Nishimura RA Steckelberg JM. Complexity and Subtlety of Infective Endocarditis. Mayo Clin Proc 2007;82:615-612.

5. Solís-Solís LD Quesada-Aguilar Carlos. Endocarditis Infecciosa en el Hospital San Juan de Dios 2000 a 2010: Parte I Agentes Etiológicos y Patrón de Resistencia Antimicrobiana. Rev ClinEsc Med UCR-HSJD. 2011;1:13-23.

6. Yock-Corrales A Segreda-Costenla A UlloaGutiérrez R. Infective Endocarditis at Costa Rica's Children's Hospital, 20002011.Pediatr Infect Dis J 2014;33:104-106.

7. Htwe HT Khardori NM. Cardiac Emergencies: infective endocarditis, pericarditis, andmyocarditis. Med Clin North Am. 2012;96:1149-1169.

8. Moreillon P Que YA. Infective endocarditis. Lancet 2004;363:139-149.

9. Beynon RP Bahl VK Prendergast BD. Infective endocarditis. BMJ 2006;333:334-339.

10. Thuny F Grisoli D Collart F Habib G Raoult D. Management of infective endocarditis: challenges and perspectives. Lancet 2012; 379: 965-975.

11. Baddour L Wilson WR Bayer AS et al. Infective Endocarditis: Diagnosis, Antimicrobial theraphy, and Management of Complications: A Statement for Healthcare Professionals From the Committee on Rheumatic Fever, Endocarditis, and Kawasaki Diesease, Council on Cardiovascular Disease in the Young, and the Councils on Clinical Cardiology, Stroke, and Cardiovascular Surgery and Anesthesia, American Heart Association: Endorsed by the Infecious Dis- eases Society of America. Circulation 2005;111:e394-e434.

12. Chu VH Park LP Athan Eet al. Association Between Surgical Indications, Operative Risk, and Clinical Outcome in Infective Endocarditis. Circulation 2015;131:131-140.

13. Kang DH Kim YJ KIM SH et al. Early Surgery versus Conventional Treatment for Infective Endocarditis. $\mathrm{N}$ Eng $\mathrm{J}$ Med 2012;366: 2466-73.

\section{CONFLICTO DE INTERÉS Y/O AGRADE- CIMIENTOS}

Los autores declaran que no existió ningún conflicto de interés en el presente reporte. 